\title{
Du disciple au maître : Victor Segalen et Paul Gauguin
}

Le peintre «sauvage » Paul Gauguin et le romancier du Divers Victor Segalen, chacun de leur côté et à des étapes diamétralement opposées de leurs vies, ont pris à cœur de dépeindre l'âme maorie pour retrouver la trace de ses origines. Le deuxième emprunte le style postimpressionniste de son gourou Paul Gauguin pour créer une œuvre littéraire à la manière d'une œuvre d'art. Dans son appréhension de la culture maorie, le patrimoine du peintre, mort en 1903 à Hiva-Oa, a été pour Segalen une introduction capitale. Lorsqu'il médite sur les travaux du peintre, Segalen institue Gauguin en médiateur dans sa démarche littéraire. Il confère à l'art de l'artiste un rôle d'initiateur et fait du peintre une sorte de prédécesseur pour l'entreprise qu'il se proposera lui-même de réaliser. En 1906, alors qu'il achève sa " prosographie " (Cachot, 1999), Les Immémoriaux, il explique qu'il a « essayé d'écrire les gens tahitiens d'une façon adéquate à celle dont Gauguin les vit pour les peindre : en eux-mêmes et du dedans en dehors » (1995, p. 103), c'est-à-dire en adoptant leur point de vue sur le monde et sur les Européens. Ainsi la peinture de Gauguin joue-t-elle un rôle central dans l'œuvre de Segalen.

\section{Paul Gauguin en tant que Maître-du-Jouir}

Un nouveau mode de représentation et de construction de l'espace, situé à l'opposé de l'esthétique naturaliste, a inspiré les poètes du début du XXe siècle et les a incités à chercher également de nouvelles formes d'expression littéraire. À titre d'exemple,

Agnieszka Kukuryk - maitre de conférences à l’Université Pédagogique de Cracovie (Chaire de littératures francophones). Adresse de correspondance : Instytut Filologii Romańskiej UP, ul. Podchorążych 2,30-084 Kraków, Pologne ; e-mail : agnieszka.kukuryk@up.krakow.pl

ORCID iD : https://orcid.org/0000-0003-1721-6820 
Guillaume Apollinaire, fasciné par certaines techniques picturales, se sert d'une dynamique spatiale pour réunir deux modalités d'expression, à savoir le discours et le graphisme. Segalen, en revanche, s'inspire des œuvres de style impressionniste de Gauguin pour créer ses Immémoriaux, livre qui révèle une tentative de dépeindre la culture maorie de l'intérieur. C'est une œuvre où l'esthétique picturale s'entremêle avec l'imagination de l'auteur et c'est pour cette raison qu'il est difficile de la classifier dans une catégorie bien définie parmi les genres. C'est à la fois un roman, une étude ethnographique, un poème ou même une confession personnelle. L'auteur lui-même préfère le terme ouvrage, mettant ainsi l'accent sur l'importance de la création. L'inspiration tirée des écrits de Gauguin y reste bien visible. Soulignons tout d'abord que c'est ce peintre qui commence le manuscrit de ses notes autobiographiques par la formule «Ceci n'est pas un livre ». Dans une lettre à son ami Fontainas, il s'explique sur la question de la façon suivante :

Ce n'est point une œuvre littéraire d'une forme choisie entre autres, c'est autre chose ; le civilisé et le barbare en présence. Là donc le style doit concorder, déshabillé comme l'homme tout entier, choquant souvent. Cela m'est facile du reste - je ne suis pas écrivain. (Manceron, 1991, p. 179)

Gauguin met ainsi en évidence l'activité de l'auteur dans la production de son œuvre, qui devrait être la synthèse de ce que l'artiste observe et de l'idée qu'il s'en fait. Rappelons tout de suite le commentaire de Victor Segalen à propos de son René Leys ${ }^{1}$ : " Je dois clore, avant de l'avoir mené bien loin, ce cahier dont j'espérais faire un livre. Le livre qui ne sera pas non plus. (Beau titre posthume à défaut d'un livre : 'Le livre qui ne fut pas' !)» (Manceron, 1991, p. 179).

Le poète est conscient de la difficulté de la création littéraire. Il oscille toujours entre le réel et l'imaginaire, ce qu'il exprime de la manière suivante :

Dans ces centaines de rencontres quotidiennes entre l'Imaginaire et le Réel, j'ai été moins retentissant à l'un d'entre eux, qu'attentif à leur opposition. - J'avais à me prononcer entre le marteau et la cloche. J’avoue, maintenant, avoir surtout recueilli le son. (Segalen, 1995, p. 318)

Pourtant, dans Les Immémoriaux, il se sert de tous les moyens artistiques pour donner une vision littéraire du peuple maori. Mais la picturalisation du texte littéraire de Segalen se manifeste non pas par le biais de la couleur, mais par un dispositif comparatif avec un tableau postimpressionniste, celui de Paul Gauguin.

1. Roman écrit à Pékin entre le 1er novembre 1913 et le 31 janvier 1914, paru en 1922, trois ans après la mort de l'auteur. Il est composé comme un journal intime dans lequel un narrateur nommé Segalen part à la recherche de l'introuvable Fils du Ciel. 
Ainsi, la démarche poétique de l'écrivain se déroule en trois étapes : la perception, la contemplation et enfin la création. La description de Segalen est toujours sensuelle, ce que Jean Starobinski constate, dans la préface des Cliniciens ès lettres, en ces termes:

Nul, sans doute mieux que Segalen, n'a su découvrir que la saisie scientifique du monde laisse insatisfait en nous le désir d'un sens, qu'il faut tenter de rejoindre par d'autres voies plus personnelles, plus démunies. (Starobiński, 1980, p. 30)

Il s'agit donc de sentir, de penser, et enfin de créer pour passer " du réel à l'art, puis par l'art, à un autre réel », selon la formule de Starobinski (p. 28). L'analogie avec la peinture de Paul Gauguin est incontestable. Segalen le souligne lui-même, en se référant au tableau le plus connu du peintre, la toile intitulée Doò̀ venons-nous? Que sommes-nous? Où allons-nous? Dans son Hommage à Gauguin, l'auteur note :

Ni blancs, ni jaunes, ni noirs, les Maoris, pour être peints, même avec des mots, ne se doivent comparer à aucune autre espèce d'hommes. [...] Il faut donc, - et le peintre s'y est magnifiquement résolu, les contempler sous leur sauvage énigme, celle qu'ils emporteront dans leur mort prévue, la question totalement humaine :

- D’ò̀ venons-nous? Que sommes-nous? Où allons-nous? (Segalen, 1995, p. 360)

L'écrivain trouve chez le peintre son alter ego qui lui apprend à faire parler l'Autre en respectant son langage et sa culture. C'est pour cette raison qu'il tente de présenter la culture tahitienne de l'intérieur comme s'il y était, ce que Henry Bouillier remarque lorsqu'il dit : « Le narrateur de l'histoire, Segalen, n'indique nulle part qu'il est lui-même maori, mais tout ce qu'il raconte, sa façon de voir et d'interpréter implique qu'il appartient à cette race » (Bouiller, 1986, p. 129).

Il ne faut tout de même pas oublier que Segalen, qui est à la fois poète, archéologue, romancier et voyageur, a, tout au long de sa vie, confronté le réel et l'imaginaire. Devenu médecin de la marine ${ }^{2}$ en 1902, il est affecté en Polynésie. Sa bonne connaissance de l'Océanie fait du poète un bon observateur de la nature pour qui « voir » est devenu une manière de goûter le monde comme un beau spectacle des apparences (Huang, 2007, p. 153). Il est persuadé qu'il existe une réalité au-delà du sensible et désire mettre sur le papier les expériences et les choses vues et contemplées. Avant de se mettre au travail, il a lu de nombreux livres sur l'Océanie. Il a été aussi bien impressionné par Salammbô de Gustave Flaubert que par Le Mariage de Loti de Pierre Loti, mais ce sont les tableaux et les écrits de Paul Gauguin qui l'ont le plus inspiré. Le 29 novembre 1903, dans une lettre à son ami Monfreid, Segalen note : "Je puis dire n'avoir rien $v u$ du pays et de ses Maoris avant d'avoir parcouru et presque vécu les croquis de Gauguin » (Segalen, 1995, p. 103).

2. Victor Segalen a soutenu sa thèse de médecine, sous le titre L'observation médicale chez les écrivains naturalistes, à l'École principale du service de santé de la marine de Bordeaux. 
La constatation du poète paraît essentielle. Étienne Barilier précise bien que l'intention du jeune écrivain n'était pas la traduction de la réalité tahitienne mais sa création (1998, p. 112). Gauguin est devenu pour lui un visionnaire qui a réussi à bondir plus loin que le Réel, « de l'autre côté de l'espace, vers le pays au-delà de tout mal, de tout bien, de toute existence manifestée » (Segalen, 1995, p. 289). C'est pour cette raison qu'il l'a placé à côté d'Arthur Rimbaud dans la série intitulée "Les Hors-la Loi » de décembre 1904, notant que pour ces deux artistes, il s'agit de faire émerger leur sensibilité individuelle dans leurs œuvres. Dès le deuxième paragraphe, l'écrivain présente Gauguin comme un homme inclassable : «Ce fut un monstre. C'est-à-dire qu'on ne peut le faire entrer dans aucune des catégories morales, intellectuelles ou sociales qui suffisent à définir la plupart des individualités. [...] il fut divers, et, dans tout, excessif » (Segalen, 1995, p. 288). Segalen a consacré aux peintures et aux sculptures de Gauguin découvertes en Polynésie deux articles : « Gauguin dans son dernier décor », publié dans le Mercure de France en 1904, et « Hommage à Gauguin », préface à l'édition des lettres de Paul Gauguin au peintre Georges Daniel de Monfreid, parue en 1916. Dans ce premier texte, le poète français trace le portrait d'un artiste démiurge qui crée dans une harmonie de tous les sens. L'art du peintre postimpressionniste n'est pas pour lui une simple transcription du réel en images mais aussi, ou avant tout, la manifestation de son affectivité par rapport au monde. À cet égard, il s'agit, comme le remarque René Huyghe, de "mettre en branle toutes ses forces sensibles et de tenter, par la contemplation, de les déceler, puis de les manifester par des lignes et des couleurs » (1986, p. 202). Segalen partage en l'occurence l'opinion du peintre, selon qui « le mot : art, appellera la reproduction de ce que les sens perçoivent dans la nature à travers le voile de l'âme " (Cachot, 1999, p. 15). Sensible au style de la peinture primitive de Gauguin, le poète désire pénétrer le monde ; sa vision ambitionne « de saisir le monde comme une réalité concrète, palpable et tangible par une expression imaginaire du réel » (Cachot, 1999, p.15). Selon Henry Bouillier, Segalen a bien senti où résidait la vérité profonde. On ne trouve aucune « mièvrerie dans ce monde pictural, ni d'anecdotes sentimentales, mais l'expression, ou plutôt, la suggestion de ce que fut l'âme maorie quand rien n'empêchait encore son dialogue incessant avec ses démons et ses dieux » (1986, p. 126). De ce point de vue, la référence à la fameuse constatation de Mallarmé : « suggérer, voilà le rêve » semble naturelle. L'aspiration de Segalen est de créer une œuvre qui, sur le plan de l'art, s'opposerait à l'esthétique académique de son époque. Il a découvert chez le peintre postimpressionniste une personnalité accomplie dans son art propre, consciente que l'art est autonome, opposée à l'art mimétique. Qu'il suffise de citer son credo, dans une lettre de 1888 écrite à Émile Schuffenecker, où Gauguin a noté :

Un conseil, ne copiez pas trop d'après nature, l'art est une abstraction, tirez-la de la nature en rêvant devant, et pensez plus à la création qu'au résultat. C'est le seul moyen de monter vers Dieu en faisant comme notre divin Maitre, créer. (1949, p. 321) 
Le peintre rêvait d'effectuer la synthèse de trois caractéristiques : l'apparence extérieure des formes naturelles, les sentiments de l'artiste sur son sujet et la pureté esthétique de la ligne, de la couleur et de la forme. Il s'est ainsi inscrit dans le mouvement littéraire et artistique de la fin du XIX ${ }^{e}$ siècle, le symbolisme, et a ouvert la voie à la peinture du $\mathrm{XX}^{\mathrm{e}}$ siècle ainsi qu'à l'art moderne. Segalen voulait poursuivre le même chemin en littérature, en transposant sur le plan littéraire la vie d'une race en voie de disparition. De même que l'artiste a peint sur ses toiles les coutumes, les croyances des Maoris comme un conservateur d'images, le poète français a cherché, à travers les livres et les derniers témoins, à saisir les traces de l'ancien royaume du bonheur. Il a expliqué son désir dans une lettre à son ami dans les termes suivants :

J'ai essayé " d'écrire » les gens tahitiens d'une façon adéquate à celle dont Gauguin les vit pour les peindre : en eux-mêmes, et du dedans en dehors. Et ce n'est pas ma moindre admiration envers lui que cette illumination de toute une race répandue dans son œuvre tahitienne. (Segalen, 1995, p. 103)

L'intention du poète de transposer dans l'ordre littéraire tout ce qu'a fait Gauguin dans l'ordre pictural, reste bien visible. Pour parvenir à son but, il a fallu comprendre toutes les démarches mises en œuvre par l'artiste.

\section{Gauguin et Segalen chez les Maoris}

Le poète et le peintre ne se sont jamais rencontrés. Pourtant, les parallélismes dans leurs biographies sont assez nombreux. Tous deux sont d'origine bretonne, mais leur histoire a commencé au printemps 1903 quand le jeune Segalen (24 ans), affecté comme médecin sur un navire de la flotte française, La Durance, a débarqué à Tahiti. À cette époque-là, le peintre, âgé de cinquante-quatre ans, vivait sur l'île de HivaOa (l'ancienne Dominique), dans l'archipel des Marquises. Segalen ne connaissait pas ses toiles avant son voyage en Polynésie. Cependant, après la mort de Gauguin le 8 mai 1903 à Tahiti, c'est le poète qui fut chargé de ramener en France les biens du peintre. Il fut donc le premier Français qui ait lu ses lettres et connu ses amis. C'est à ce moment que Segalen a découvert dans les tableaux de Gauguin la population polynésienne qui vivait en symbiose avec le milieu naturel. Il est devenu l'épigone du peintre et, comme lui, il a d'abord pénétré l'âme des Maoris pour ensuite la décrire. Comme le précise bien Laurence Cachot, le poète " fait parler l'Autre » (1999, p. 15). Ce n'est plus l'image de l'Autre issue des œuvres sur l'exotisme de Montesquieu ou de Diderot, l'Autre qui restait l'étranger des îles lointaines. Dans Les Immémoriaux, c'est l'Européen qui devient l'Autre aux yeux des Polynésiens natifs, et aux yeux de Segalen qui tente de transmettre cette conception de l'Autre au lecteur. Cette remarque est mise en exergue par Bouillier : 
Les missionnaires étrangers "à peau blême ", leurs coutumes, leurs cultes sont bien décrits et raillés sous l'angle maori, et dans ce livre composé par un Européen, ce qui apparaît exotique, au sens banal du mot, c'est non pas le mode de vie indigène, mais celui des arrivants. (Bouillier, 1986, p. 130)

L'Autre et l'Ailleurs agissent alors comme un jeu de miroir dans lequel l'auteur interroge son propre reflet, au point que Segalen déclarera en 1929 : « Je pars, je m’agite dans l'espoir seulement du retour enrichi » (1983, p. 18). Ce que Segalen voit dans l'Autre, ce n'est pas son existence propre, mais sa différence. Entre soi et le monde, entre soi et l'Autre résonne alors une différence profonde, mêlée d'absence :

Ainsi, l'on ne tentera point, [dit-il] après tant d'autres, une nouvelle ébauche étrangère de leurs contours, de leurs aspects, de leurs coutumes, et des gestes de leurs cultes. On ne veut point cataloguer leurs armes ni leurs objets familiers; on ne mettra point leurs dieux en vitrines, et il ne sera pas, pour tout dire, question d'anthropologie, ou d'exégèse, ou d'ethnographie polynésiennes [...]. (Segalen, 1995, p. 383)

Segalen joue donc le rôle d'un membre des Maoris et tente de "penser en leur place ", de " raconter selon leur guise » (Segalen, 1995, p. 383). Suivant le chemin de son maître Gauguin, qui s'est adapté aux canons esthétiques de Tahiti, il essaie dans son œuvre d'inventer la forme littéraire qui pourrait pénétrer avec plus de succès dans une culture aux prises avec la mort et l'oubli.

En dépit de la divergence des techniques existant entre la littérature et la peinture, Segalen a tenté de créer dans les Les Immémoriaux un effet d'écriture comparable à celui de l'art. C'est pour cette raison qu'il a oublié toute divergence et s'est référé à toutes les techniques accessibles de l'art plastique. Pour réussir, il a profité de tous les moyens du langage poétique afin de réaliser la picturalité. L’analogie entre les figures décrites par Segalen et celles des toiles de Paul Gauguin permet de considérer que cet objectif n’était pas hors de portée.

\section{Poésures polynésiennes de Segalen ${ }^{3}$}

Les Immémoriaux se composent de trois parties qui relatent l'histoire de la décadence du peuple tahitien. La première décrit la société polynésienne avant l'arrivée des Européens et des missionnaires, la deuxième constitue une période de transition que Segalen définit comme « la part nautique et légendaire... Sa raison dêtre

3. Nous nous référons ici à l'exposition "Poésure et peintrie " conçue par Bernard Blistène au Musée de Marseille en 1993 qui racontait l'aventure menant la littérature à explorer ses expressions matérielles, visuelles et sonores, depuis la spacialisation mallarméenne du texte. (Bernard Blistène et al., Poésure et peintrie : d'un art, l'autre, cat. exp., Centre de la Vieille Charité, Marseille, 12 févr.-23 mai 1993, Marseille / Paris, Réunion des musées nationaux). 
dans le récit est de [..] faire gagner vingt ans, et aussi d'appuyer sur les anciens Dires une dernière fois » (Segalen, 1995, p. 104). La dernière partie présente la même société après vingt ans de soumission au christianisme européen.

Puisque l'intention de l'auteur était de faire un livre à la façon d'une œuvre d'art, il a renoncé à la tradition psychologique très perceptible dans la littérature française de l'époque. Les personnages sont donc dépourvus de sentiments, il n'y a pas non plus de portraits individuels mis en valeur. Le héros central du livre, c'est le peuple tahitien tout entier. Il y a de l'avis général des personnages dits romanesques comme Paofaï, le païen obstiné ou comme son fils Térii élevé pour être le futur continuateur des anciens cultes, mais le premier n'a pas de caractère complexe et le deuxième représente plutôt l'ensemble des traits qui caractérisent le peuple à l'heure de la décadence.

Cependant, l'objectif de Segalen était avant tout de mettre en relief la beauté tahitienne, de la faire sentir. C'est sans doute pourquoi les descriptions des femmes maories paraissent comme le pendant de celles que le peintre a mises dans ses toiles. D'après Henry Bouiller, le poète a emprunté la vision esthétique de Gauguin à tel point que « la description par Segalen de la femme maorie se présente d'abord comme un simple commentaire des personnages du peintre» (1986, p. 474). Pour Segalen, Tahiti semble un territoire sacré où la nature est enceinte de signes où, «à travers les visages, [dit-il], pénétraient jusqu'au fond des poitrines, les formes familières des monts, le grand arc de corail, la couleur de la mer, et la limpidité des favorables firmaments » (1995, p. 160). Selon le poète, la beauté n'a pas besoin d'ornement superficiel. Il souligne ainsi son admiration pour les femmes maories qui « avaient tout le corps enroulé de tapa : et cela qui doublait leur embonpoint, les rendait plus désirables" (p. 138). La nature avec laquelle le corps féminin se confond souvent est d'ailleurs le seul ornement que Segalen admet : « Les oreilles d'Éréna se paraient de fleurs rouges, ouvertes, elles-mêmes comme d'autres oreilles parfumées. Ses cheveux étaient ceints, de feuilles menues et odorantes aussi » (p. 216). D'ailleurs l'auteur, se servant des formes primitives de Gauguin, cherche à faire voir les femmes avec précision. De la même façon que le peintre esquisse les Polynésiennes en gros plan, il les met en valeur. De plus, toutes les descriptions du corps sont pleines de sensualité : « Les doigts courbés rampaient autour de son cou, effleuraient la gorge et la nuque, enfermaient l'épaule ronde, et se glissant dans l'aisselle, venaient, à travers l'étoffe limpide, presser le versant du sein » (p. 189). Parfois, il anime le corps de la femme ou colore certaines scènes - pour faire entrer le spectateur dans son spectacle : " Les filles tournoyaient sur elles-mêmes. Les nattes longues démesurément se déroulaient en changeant de couleur : blanches, rouges, blanches et rouges encore" (p. 139). Ce mouvement, comme le remarque Cachot, " provoque une impression de prolongement infini de la femme " (1999, p. 24). Puisque l'image chez l'écrivain est essentiellement la peinture, sa plume prend en charge une description qui doit donner l'équivalent du visuel. Les mots comme les coups de pinceau se précipitent pour donner une image picturale. Le poète invite le lecteur à donner consistance à un tableau littéraire issu de son imagination. Un tableau surgit ainsi dans la texture de ce récit. 
Notons aussi que Segalen s'inspire également de l'écriture visuelle de la culture étrangère. Dans le manuscrit du livre, l'écriture avoisine le dessin, il y a beaucoup de bulles, de ratures, d'encadrements. Ces formes, lettres, chiffres, qu'Étienne Germe appelle des "événements graphiques ", situés dans la marge, constituent une écriture parallèle et sont un prolongement du texte littéraire (2001, p. 115). À cet égard, l'auteur tatoue son écriture à la manière de la culture tahitienne et donne quelques exemples où les chiffres et les lettres s'insinuent dans un dessin, ce qui donne l'impression d'une écriture primitive.

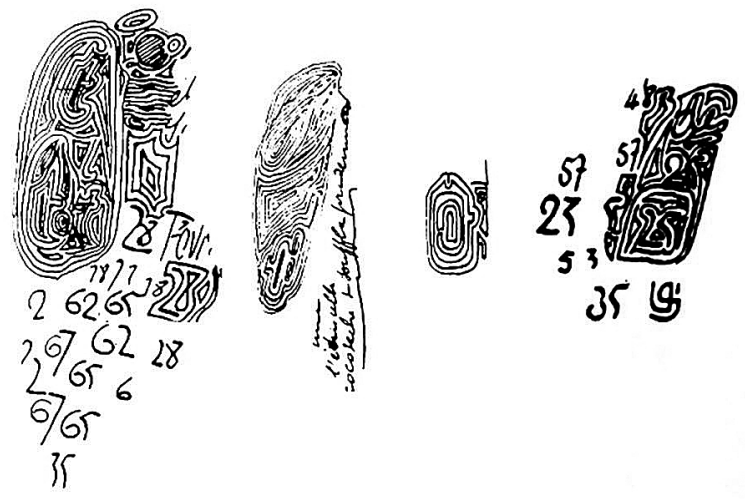

Les Immémoriaux I (p. 8, 47, 49, 58)

Segalen joue aussi avec la forme en s'inspirant des signes des Maoris. À l'instar de ceux-ci, il crée ses propres symboles et les place soit en marge soit au centre du texte. L'écriture océanique, qui prend souvent la forme du tatouage, est pour lui la marque d'une identité, de toute une histoire. Les linéaments, dans la première version des Immémoriaux, ne sont pas le résultat d'un hasard ni d'une distraction de la part de l'auteur. Ce dernier a très bien examiné l'aspect sociologique de « ces signes noirs » et leur importance pour les habitants des îles polynésiennes, ce qu'il explique de la façon suivante :

Quant aux signes, on les tatoue, avec une pierre courante, sur des bois polis et plats qu'on nomme ensuite Bois-intelligents. Lorsque la tablette est incrustée comme une peau de chef, alors l'homme habile y trace son Rua, qui est sa marque à lui-même.

Et l'on peut, longtemps après, reconnaître un à un les signes - comme un homme reconnaît ses fétii - par leurs noms. On dit alors : les Bois parlent. (Segalen, 1995, p. 178)

L'intention de Segalen était de suivre la même démarche, mais en fin de compte, il a compris que ce ne serait qu'un calque qui n'apporterait aucun sens nouveau. Il a donc abandonné l'idée de mettre les mots en image, idée à laquelle il reviendra cependant quelques années plus tard. Dans la version finale du livre, le poète a renoncé 
à l'aspect visuel des signes des îles dans le texte. Il a malgré tout gardé un symbole au début des Immémoriaux et l'a enrichi d'une constatation cosmogonique : « La terre et l'homme».

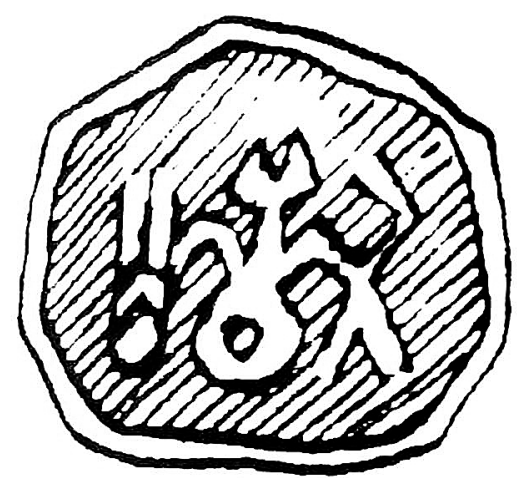

Dans les versions ultérieures, le lecteur peut retrouver ce pictogramme sur la reliure du livre ; le pictogramme en question est placé à côté d'une idole dessinée par Paul Gauguin comme si ces deux images traduisaient le même mystère.

Les Immémoriaux constituent le portrait du peuple maori qui oublie ses mots et ses dieux. S'inspirant de nouvelles formes artistiques, en particulier du style postimpressionniste de Gauguin, Segalen veut donner par écrit l'équivalent de ce qu'on pourrait voir si l'on se tenait devant un tableau. Il désire évoquer des peintures imaginaires, ou bien organiser l'écriture de telle façon qu'il y ait visualisation de l'écrit. À partir des mots, le poète fait de son texte un tableau imaginaire. Il crée au moyen des mots une image visuelle, tout en mettant entre les mains du lecteur un tableau accessible. De ce fait, Segalen exalte la puissance du langage et de l'écriture.

\section{En guise de conclusion}

Le séjour à Tahiti confirme les ambitions littéraires du poète et l'encourage à chercher une forme originale pour ses futures œuvres. Un an après son retour en France, en février 1906, dans une lettre à son ami Charles Guibier, il écrit : " Je suis né pour vagabonder, voir et sentir tout ce qu'il y a à voir et sentir au monde. Je poursuivrai ma collection. À commencer sans doute par l'Extrême-Orient »(Segalen, 1995, p. 5). Les poèmes de Stèles, écrits entre 1910 et 1912, justifient sa fascination pour l'expression verbale et l'expression graphique ainsi que pour la représentation de l'espace de la page. Cependant, grâce aux Immémoriaux, Segalen devient l'auteur d'une œuvre qui puise dans l'exotisme ses légendes et ses décors. Son premier voyage en Polynésie marque le début du Segalen écrivain, hanté par la présence récente d'un peintre français lui aussi en quête d'un Ailleurs : Gauguin. Celui-ci est devenu son père spiri- 
tuel dans la composition d'une œuvre qui dépasse le cadre d'un récit de voyage. Ici, le narrateur analyse tout en poursuivant un long travail sur l'écriture. L'influence de la vision de Gauguin, que Segalen nomme Maître-du-Jouir, sur la création littéraire de l'écrivain est fortement visible. Dans Les Immémoriaux, le poète effectue une sorte de traduction d'un art à l'autre qui est pour lui une démarche créatrice par elle-même.

\section{RÉFÉRENCES}

Barilier, É. (1998). Segalen, Gauguin, Debussy. Dans Marie Dollé et al. (dir.), Victor Segalen (p. 111-116). Paris : Éd. de l'Herne.

Bouillier, H. (1986). Victor Segalen. Paris : Mercure de France.

Cachot, L. (1999). La femme et son image dans l'ouvre de Victor Segalen. Franche Comté : Annales Littéraires de l'Université de Franche Comté, nº 689.

Gauguin, P. (1949). Lettre à Émile Schuffenecker [1888]. Dans M. Malingue (éd.), Paul Gauguin, Lettres à sa femme et à ses amis. Paris : Grasset.

Germe, É. (2001). Segalen, l'écriture, le nom. Architecture d'un secret. Saint-Denis : Presses Universitaires de Vincennes.

Huang, B. (2007). Segalen et Claudel. Dialogues à travers la peinture extrême-orientale. Rennes : Presses Universitaires de Rennes.

Huyghe, R. (1986). Gauguin. Paris : éd. du Chêne.

Manceron, G. (1991). Segalen. Paris : Jean-Claude Lattès.

Segalen, V. (1975). Gauguin dans son dernier décor et autres textes de Tahiti. Paris : Fata Morgana.

Segalen, V. (1983). Équipée. Paris : Gallimard.

Segalen, V. (1995). Euvres complètes. Tome 2. Paris : éd. Robert Laffont.

Starobinski, J. (1980). Les Cliniciens ès lettres. Montpellier : Fata Morgana.

RÉSUMÉ : L'idée de cet article vise à présenter la relation particulière entre le peintre et le poète et à examiner l'influence de la vision de Gauguin, que Segalen nomme le Maître-du-Jouir, sur la création littéraire de l'écrivain. Les deux Français avaient beaucoup en commun : le sens du mystère, une fascination pour le primitif et l'exotique et un don pour l'ambiguiité. À partir de 1903 jusqu’à sa mort en 1919, la poésie et la prose de Segalen tenteront, à l'instar de l'art de Gauguin, de découvrir l'exotisme à leur manière tout en se confrontant de plus en plus aux limites du pouvoir d'appréhension de l'artiste occidental. Dans Les Immémoriaux, Segalen adopte un point de vue maori pour commenter la culture occidentale. Le plus grand exploit de l'auteur est de rompre avec l'exotisme colonial des écrivains voyageurs du XIX ${ }^{e}$ siècle, par exemple Pierre Loti. Là où ces auteurs ne prétendaient pas renoncer aux valeurs occidentales, voire impériales, tout en décrivant des cultures étrangères, Segalen, imitant son maître Gauguin, était extrêmement conscient de ses représentations de l'autre de manière particulièrement moderne. Segalen réalise dans son roman 
ethnographique une sorte de translation d'un art à l'autre qui est pour lui une démarche créatrice par elle-même.

Mots-clés : Les Immémoriaux, Victor Segalen, Paul Gauguin, exotisme, culture maorie

\section{From the disciple to the teacher: Victor Segalen and Paul Gauguin}

ABSTRACT: The aim of this paper is to present a particular relationship between the painter and the poet, and to examine the influences of Gauguin's vision that Segalen calls Maitre-du-Jouir on the literary creation of the writer. The two Frenchmen had much in common: a sense of mystery, a fascination with the primitive and the exotic, and a gift for ambiguity. From 1903 until his death in 1919, Segalen's poetry and prose would, like Gauguin's art, try to discover the exotic on its own terms while increasingly confronting the limits of the Western artist's own powers of apprehension. In Les Immémoriaux (translated into English as A Lapse of Memory), Segalen adopts a Maori point of view to comment on Western culture. The author's greatest feat was breaking with the colonial exoticism of such nineteenth-century writer-travelers as Pierre Loti for example. Where those authors made no pretence about surrendering Western, or even imperial, values while depicting foreign cultures, Segalen, imitating his Master Gauguin, was intensely self-conscious about his depictions of the other in ways that seem especially modern. In his ethnographic novel, Segalen performs a kind of translation from one art to another which is, for him, a creative process by itself.

Keywords: Les Immémoriaux, Victor Segalen, Paul Gauguin, exoticism, Maori culture 